\title{
Notas sobre ¿Error o fraude?: libro póstumo de Ernesto Pollitt
}

\section{Notes on Error or Fraud? Posthumous Book of Ernesto Pollitt}

\section{Tomás Caycho Rodríguez}

Universidad Privada del Norte, Lima, Perú.

Recibido: $19-07-16$

Aprobado: 29-08-16

\section{Correspondencia}

Email: tppcaycho@gmail.com

\section{Citar como:}

Caycho, T. (2016). Notas sobre ¿Error o fraude?: libro póstumo de Ernesto Pollitt. Propósitos y Representaciones, 4(2), 413-426. Doi: http://dx.doi.org/10.20511/ pyr2016.v4n2.126

(C) Universidad San Ignacio de Loyola, Vicerrectorado de Investigación y Desarrollo, 2016. (๕) BY.NC-ND Este artículo se distribuye bajo licencia CC BY-NC-ND 4.0 Internacional (http://creativecommons.org/licenses/by-nc-nd/4.0/). 
Pollitt, E. (2016). ¿Error o fraude? Engaños y equivocaciones en la investigación psicobiológica infantil. Lima: Fondo Editorial de la Pontificia Universidad Católica del Perú. 198 págs. ISBN: 978-612-317-170-4

El año 2016 se inicia con una lamentable noticia para la comunidad psicológica peruana e internacional: la muerte del Dr. Ernesto Pollitt Burga, considerado uno de los psicólogos peruanos más importantes en nuestro país y el de mayor renombre internacional (Arias, 2016; Caycho, 2016; Raez, 2016), especialista en diversos temas relacionados con el desarrollo infantil, como los efectos de la alimentación y malnutrición en el desarrollo y la conducta infantil. Meses después de su fallecimiento, la Pontifica Universidad Católica del Perú (PUCP), en donde Pollitt siguió los estudios de Psicología, a través de su fondo editorial, acaba de publicar de manera póstuma el último libro del afamado psicólogo nacional, titulado ¿Error o fraude? Engaños y equivocaciones en la investigación psicobiológica infantil, el cual se ocupa de un importante aunque escasamente comprendido tema: la formulación de políticas sociales, basadas en evidencias científicas válidas y confiables, destinadas a mejorar la salud y las condiciones de vida de la infancia.

A pesar de lo trascendente del tema, y salvo la reseña realizada por Cueto (2016), la publicación de la obra ha pasado relativamente desapercibida en los ámbitos académicos peruanos. El libro reúne una gran cantidad de información de suma utilidad para quienes estamos inmersos en la investigación científica sobre el desarrollo infantil, y se encuentra basado en la experiencia del autor a lo largo de intensos años de trabajo de campo, observaciones clínicas e intervenciones acerca de las condiciones sociales y psicológicas de la niñez en el Perú y el mundo.

El libro está conformado por un prólogo, una introducción, seis capítulos y un glosario de términos. En el prólogo, la Dra. Susana Frisancho, catedrática de la PUCP, muy acertadamente señala que el texto de Pollitt "llena, sin duda, un vacío en la literatura especializada en investigación psicológica en 
nuestro país" (p. 13). No podríamos estar más de acuerdo pues el tema del fraude y los errores en la investigación psicológica, y por qué no decirlo en otras ciencias también, se encuentra latente a cada momento.

La introducción de Pollitt no es solamente un breve resumen de lo que el lector podrá encontrar en el libro; es, ante todo, una expresión de los lineamientos teóricos y metodológicos seguidos por el autor para hacer los análisis y llegar a las conclusiones de cada capítulo. Aquí, y desde un inicio, Pollitt menciona el tema principal de todo el libro al escribir que:

Idealmente, las políticas y regulaciones sociales, así como las prácticas profesionales dirigidas a beneficiar la calidad de vida de una persona, de un grupo o de una sociedad, deben estar basadas en datos válidos y confiables (evidencia) generados a través del método científico (p. 17).

Así, en esta introducción, el autor pasa revista a conceptos, como el de desarrollo psicobiológico, plasticidad, y a metodologías, como la investigación controlada o ensayo clínico, sus beneficios y limitaciones, la ética en los diseños experimentales, las limitaciones del empleo de escalas para evaluar el desarrollo mental, los tipos de errores en la decisión sobre una hipótesis, conceptualizado por Pollitt como "aquellas situaciones en las que el autor no ha tenido ni intención ni conciencia del error” (p. 22), y su diferencia y relación con el fraude que, aunque es difícil de definir, es común asociarlo con "fabricación, falsificación y plagio de información" (p. 22). Así también, en esta introducción, Pollitt da cuenta de la importancia de analizar las diversas presiones personales, institucionales, económicas y políticas que podrían llevar a un científico a cometer un fraude.

El primer capítulo analiza dos casos respecto del fraude y el error. El primero describe el caso del bioquímico Ranjit Chandra y su investigación sobre el deterioro cognitivo en la vejez y los beneficios de una serie de suplementos vitamínicos y minerales. Varias publicaciones dieron cuenta de los errores metodológicos del estudio, llevando a la revista Nutrition, donde originalmente se publicó el estudio de Chandra, a retractarse de la 
publicación de la mencionada investigación aduciendo pobreza en las réplicas de Chandra a sus críticos, dudas sobre la validez de otras publicaciones e interés económico acerca del suplemento de vitaminas y minerales. E1 segundo caso, conocido mundialmente en el campo de la psicología, es el de Cyril Burt, quien fue acusado de haber fabricado los datos de su estudio. Todo esto llevó a una serie de controversias que duraron más de 20 años luego de la muerte del psicólogo inglés.

En el segundo capítulo, Pollitt analiza uno de los casos más discutidos dentro de la psicología: John Money y la reasignación de sexo de David Reimer. Se analizan los postulados teóricos de Money en relación con el desarrollo humano, la identidad de género y la influencia sobre estos del ambiente y el aprendizaje social. Luego se describe el caso de David Reimer y las críticas al trabajo de Money que provocaron en David el padecimiento de problemas psicológicos. Pollitt concluye que:

Las decisiones institucionales de Johns Hopkins, al permitir la reasignación sexual de Bruce, se basaron en una proposición de dudosa validez sobre la determinación ambiental en el desarrollo psicosexual. La academia de Johns Hopkins fue culpable de un error de juicio tan serio -o más- que el propio error de John Money (p. 60).

El tercer capítulo centra el análisis en un caso latinoamericano: el Ministerio para el Desarrollo de la Inteligencia en Venezuela, liderado por Luis Alberto Machado, abogado, poeta, humanista y filósofo. Pollitt concluye en este acápite que el macroproyecto venezolano fue instaurado en base a un inadecuado empleo de información científica junto con objetivos más que todo de carácter político y económico.

El cuarto capítulo enfatiza las relaciones entre la biología y las características socioculturales a partir del análisis de un estudio llevado a cabo en Zanzíbar (África) sobre la influencia de la administración de hierro en la disminución de los porcentajes de repitencia y deserción de los estudiantes de la comunidad. De la lectura del capítulo se puede concluir, en consonancia con lo mencionado por Pollitt, que tanto los factores 
biológicos como socioculturales y del ambiente biofísico influyen en el desarrollo infantil.

El tema de los parásitos intestinales y su relación con el rendimiento cognitivo y académico a través de mecanismos biológicos, como la pérdida de hemoglobina y micronutrientes en el cerebro, es tratado en el quinto capítulo. Pollitt analiza los beneficios de los tratamientos antihelmínticos sobre "el desarrollo cognitivo y el aprovechamiento escolar" (p. 151) apoyados por organismos internacionales, tratando de responder a la pregunta: “QQué datos científicos atestiguan dichos efectos?” (p. 151). A partir de la revisión concienzuda y meticulosa de un conjunto de investigaciones y reportes oficiales de organismos internacionales, como el Banco Mundial, la Organización Mundial de la Salud y UNICEF, Pollitt concluye que la información derivada de las fuentes científicas e institucionales es discrepante, lo que lleva a afirmar que no existe evidencia que justifique la implementación de programas de desparasitación en población infantil. El mismo autor señala que:

En mi opinión, el Banco Mundial y la OMS, basándose en proposiciones teóricas, mas no empíricas, llegaron a conclusiones apresuradas que condujeron a la puesta en marcha de programas de desparasitación en varios países con un pobre ingreso per cápita. ¿Fraude? (p. 171).

El capítulo seis da a conocer las principales conclusiones del estudio. Aquí, Pollitt hace uso de la información derivada de cada uno de los capítulos para formular un modelo teórico acerca de los factores que influyen en la calidad de la información obtenida en los estudios científicos. Aquí podemos mencionar, en primer lugar, las fases de la investigación científica (la teoría elegida, la formulación de las hipótesis, así como el diseño de investigación y la interpretación de los datos), las demandas cognitivas del investigador y las presiones personales, institucionales, políticas económicas y culturales, las cuales pueden llevar a cometer errores durante el proceso de investigación que, a su vez, como señala Pollitt, "puede llevar a la creación 
de intervenciones sociales que afectan el desarrollo psicobiológico infantil" (p. 178).

El libro finaliza con un glosario de algunos términos aparecidos en el mismo y que son de gran apoyo para la comprensión cabal de los conceptos y análisis surgidos de la lectura de la obra. De singular importancia e interés son las secciones bibliográficas de cada uno de los capítulos, que cuentan con numerosas referencias acerca de trabajos experimentales sobre desarrollo psicobiológico infantil y los factores que influyen en él.

El libro que acabamos de revisar no puede ser considerado una crónica fría de los acontecimientos que en él se encuentran; más bien, es un texto que agita los corazones de los lectores planteándoles numerosas interrogantes acerca de lo mucho que hay aún por hacer en materia de políticas sociales a favor de la infancia, no solo en el Perú, sino también en muchos países latinoamericanos. El libro, de singular naturaleza en nuestra bibliografía psicológica peruana, creemos que servirá para un análisis diferente cuando se traten temas sobre la infancia y lo que podemos hacer por ella.

La calidad de la obra radica en su objetivo y amplitud temática, así como en la erudición de su autor, pues Pollitt trata de brindar al lector una imagen lo más completa posible de la importancia de la investigación científica seria, lográndolo a través de un estilo académico adecuado que retiene las mentes que ingresen a su lectura.

¿Error o fraude? Engaños y equivocaciones en la investigación psicobiológica infantil es una lectura recomendable para todo aquel profesional de la salud que desee conocer acerca de la formulación de políticas sociales a favor de la infancia basadas en evidencia. La selección de cada uno de los capítulos es apropiada, y la introducción concede un gran valor teórico y metodológico a la obra. 


\section{Referencias}

Arias, W. L. (2016). Ernesto Pollitt y su contribución a la psicología evolutiva en el Perú: sus investigaciones en nutrición, cognición y rendimiento escolar. Revista de Psicología, 34(2), 481-500.

Caycho, T. (2016). In Memoriam: Recordando a Ernesto Pollitt Burga (19382016). Propósitos y Representaciones, 4(1), 385-396. http://dx.doi. org/10.20511/pyr2016.v4n1.94

Cueto, S. (2016). Pollitt, Ernesto (2016). ¿Error o fraude? Engaños y equivocaciones en la investigación psicobiológica infantil. Lima: Fondo Editorial de la Pontificia Universidad Católica del Perú. 198 pp. Revista de Psicología, 34(2), 501-503.

Raez, M. (2016). Semblanza del Dr. Ernesto Pollitt Burga. Revista de Psicología, 34(2), 473-479. 